\title{
On Quantum Field Theory in Gravitational Background
}

\author{
Rudolf Haag, Heide Narnhofer*, and Ulrich Stein \\ II. Institut für Theoretische Physik, Universität Hamburg, D-2000 Hamburg 50, \\ Federal Republic of Germany
}

\begin{abstract}
We discuss quantum fields on Riemannian space-time. A principle of local definiteness is introduced which is needed beyond equations of motion and commutation relations to fix the theory uniquely. It also allows us to formulate local stability. In application to a region with a time-like Killing vector field and horizons it yields the value of the Hawking temperature. The concept of vacuum and particles in a non-stationary metric is treated in the example of the Robertson-Walker metric and some remarks on detectors in non-inertial motion are added.
\end{abstract}

\section{Introduction}

In the past decade there has been increasing interest in the interplay between the principles of general relativity and those of quantum physics. Some aspects arise already when one considers local quantum physics in a Riemannian space whose causal structure is described by a given, classical metric field $g_{\mu v}(x)$. Typical examples are the Hawking temperature of a black hole [1], the behaviour of accelerated detectors as discussed by Unruh [2] and the definition of particle states in an expanding universe. The essential phenomena and problems arising from the deviation of $g_{\mu v}$ from the Minkowski metric can be illustrated in these examples by quantum fields obeying linear field equations. We shall confine our discussion to this.

The first question is whether the quantum theory is unambiguously defined by the equation of motion and commutation relations without recourse to a special, distinguished state, the vacuum [2a]. In the Minkowski world this special state is tied to the time translation symmetry of the theory, a feature which is lost in general (there will usually be no global time-like Killing vector fields). To answer this question we have to make clear what we mean by "the theory." Our customary

* Permanent address: Institut für Theoretische Physik, Universität Wien, Wien, Austria 
description of phenomena in physics distinguishes between the "laws of nature" and "initial conditions." The theory concerns the former and leaves a large arbitrariness in the latter. In the algebraic approach to quantum physics the "laws" correspond to the fixing of an algebra (or rather a net of algebras associated with space-time regions [3]), the "initial conditions" correspond to the choice of a state (expectation functional) over this algebra. However, there is some leeway because the equations of motion and commutation relations of the field still allow a variety of choices of the algebra of bounded observables and hence of the allowed states. It appears to us a good principle, supported by all experience with quantum field theory in Minkowski space as well as by the spirit of the principle of locality, that for a small contractible neighborhood of a point the theory must fix the algebra uniquely in such a way that no superselection rules between the allowed "partial states" of such a small region ${ }^{1}$ exist. In other words, the laws should not allow inequivalent representations of the algebra of bounded observables of such a space-time region. All superselection rules are either of global nature (such as the total matter content of the universe) or of topological nature such as charge quantum numbers which have no significance for a small, contractible part of space-time. Mathematically it means that the algebra of a compact, contractible region shall be a $W^{*}$-factor, i.e. isomorphic to an operator algebra with trivial center on a Hilbert space and that only normal states of this algebra are allowed. We shall call this the principle of local definiteness. This indicates incidentally that the set of (partial) states is more fundamental than the algebra, the latter being the dual space of the linear hull of the former. Compare Sect. C of [4].

The next observation is that the usual algebraic relations written down in quantum field theory, i.e. the commutation relations and the equations of motion, are not enough to implement the laws as demanded by the principle of local definiteness. One reason for this is the following. Consider a sequence $\mathscr{O}_{n}$ of simple ${ }^{2}$ space-time regions shrinking to a point $x$ as $n \rightarrow \infty$, and in each region pick an observable $A_{n}$. The sequence $A_{n}$ will move into the commutant of the total algebra as $n \rightarrow \infty$ because there are no observables at a single point; the observables in the space-like complement of a single point generate the whole algebra. Hence any limit point of such a sequence is in the center and should be a multiple of the identity by local definiteness. For a quantum field $\Phi$ with linear field equations this problem may be reduced to the discussion of the product $\Phi\left(x_{1}\right) \Phi\left(x_{2}\right)$ with $x_{i} \in \mathscr{U}$, where $\mathscr{U}$ is a small neighborhood of the point $x$. We have to specify the singularity of the product of fields as $x_{1} \rightarrow x_{2}$. In particular, considering $\Phi\left(x_{1}\right) \Phi\left(x_{2}\right)$ as an operator-valued distribution over $\mathscr{U} \times \mathscr{U}$ we can reduce it to a distribution over $T_{x} \times T_{x}$, where $T_{x}$ is the tangent space at a point $x$ :

$$
\begin{gathered}
w_{x}\left(f^{(1)}, f^{(2)}\right)=\lim _{\lambda \rightarrow 0} \int \Phi\left(x+y_{1}\right) \Phi\left(x+y_{2}\right) f_{\lambda}^{(1)}\left(y_{1}\right) f_{\lambda}^{(2)}\left(y_{2}\right) d \mu\left(y_{1}\right) d \mu\left(y_{2}\right), \\
d \mu(y) \sqrt{-g(x)} d^{4} y,
\end{gathered}
$$

by taking a suitable sequence of test functions $f_{\lambda},\left(\left.f_{\lambda}\right|_{\lambda=1} \in \mathscr{D}(\mathscr{M})\right)$, whose support contracts to the origin as $\lambda \rightarrow 0$. For a free scalar field in Minkowski space such a

1 By "partial state" we mean the restriction of the expectation functional to the algebra of the region

2 Open, contractible regions with compact closure 
sequence is

$$
f_{\lambda}(y)=\lambda^{-3} f\left(\lambda^{-1} y\right) .
$$

The vacuum expectation value of $w_{x}$ becomes then

$$
\left\langle w_{x}\left(f^{(1)}, f^{(2)}\right)\right\rangle_{0}=\int f^{(1)}\left(y_{1}\right) D^{(+)}\left(y_{1}-y_{2}\right) f^{(2)}\left(y_{2}\right) d^{4} y_{1} d^{4} y_{2},
$$

with

$$
\begin{aligned}
D^{(+)}(z) & =(2 \pi)^{-3} \int e^{i\left(\mathbf{p z}-p^{0} z^{0}\right)} \delta\left(p^{0^{2}}-\mathbf{p}^{2}\right) \theta\left(p^{0}\right) d^{4} p \\
& =(2 \pi)^{-2}\left(z^{2}-\left(z^{0}-i \varepsilon\right)^{2}\right)^{-1}
\end{aligned}
$$

Note that $\left\langle w_{x}\right\rangle$ does not contain the mass value anymore because only the most singular part of $\Delta^{(+)}(z, m)$ survives in the limit (i.1).

The essential point is now that the principle of local definiteness implies that $\left\langle w_{x}\right\rangle_{\omega}$ of (1.1) shall be the same for all allowed states $\omega$, i.e. the operator valued distribution (1.1) is actually a numerical-valued distribution whose specification is part of the definition of the theory. This remark can be carried over almost without change now to the case of field theory in Riemannian space-time. In fact we shall see that $w_{x}$ and $w_{x}^{(n)}$ (see the next section) are uniquely prescribed by requirements of continuity in $x$, covariance under parallel transport, fixing of the scaling factor needed in (1.2) which follows from the commutation relations or the "dimension of the field," "local stability" which gives the proper $i \varepsilon$-prescription for the singularity on the light cone or, alternatively speaking, replaces the "positive energy" condition of the Minkowski theory and finally the positivity of the set $w_{x}^{(n)}$ considered as a linear form over the tensor algebra of test functions in tangent space.

The specification of the $w_{x}^{(n)}$ is necessary to fix the local $W^{*}$-algebras. It is in general not sufficient for that purpose (see Sect. IV). Once the local $W^{*}$-algebras are fixed the global algebra is naturally defined as the norm closure of the algebra generated by all the algebras of compact, contractible regions. This is a $C^{*}$-algebra which will have many inequivalent representations whose restrictions to local regions will, however, all be equivalent. The distinction between the inequivalent global representations result on the one hand from differences in the asymptotic behaviour of states at space-like infinite. On the other hand they may result from topologically different possibilities of patching together local states as in the case of charge quantum numbers (superselection sectors) known in the Minkowski-space quantum field theory. There arises however one new feature in the Riemannian case: The given metric field may describe only part of the manifold such as the outside region of a Schwarzschild hole. In that case the horizon plays at first sight the same rôle as infinity for the $C^{*}$-algebra of the accessible region. This algebra will allow representations differing in the behaviour of states on the horizon. If we know, however, that the manifold extends beyond the horizon, then the condition for the state in the tangent spaces along the horizon has to be taken into account and this gives a restriction on the allowed representations. In other words it is necessary to make the algebra of a closed simple region including points of the horizon again into a $W^{*}$-factor.We shall discuss this mechanism and its relation to the Hawking temperature in Sect. III. 
Another circle of questions is: Does there exist a reasonable definition of local vacuum and local particle states if the metric is not stationary. We shall address ourselves to this in the example of the Robertson-Walker metric in Sect. IV. Related to this is the problem of how to define detectors. We make some remarks on this in Sect. V.

\section{The Wightman Distributions in Tangent Space. Covariance and Local Stability}

In the Wightman frame the requirement of local definiteness demands that we specify the ((numerical-valued) distributions in $T_{x} \times T_{x} \times \ldots T_{x}$ arising from

$$
w_{x}^{(n)}(f)=\lim _{\lambda \rightarrow 0} \int \Phi\left(x+y_{1}\right) \ldots \Phi\left(x+y_{n}\right) f_{\lambda}\left(y_{1}, \ldots, y_{n}\right) d \mu\left(y_{1}\right) \ldots d \mu\left(y_{n}\right)
$$

for appropriate choices of the sequence of test functions $f_{\lambda}$. For an interacting field this should be done following the lines of the Wilson-Zimmermann expansion. In this paper we are concerned only with free fields where a unique answer follows from a few general properties.

For quantum fields living on a Riemannian manifold $\mathscr{M}$ we first have to convince ourselves that $w_{x}^{(n)}$ as defined by (2.1) is independent of the choice of the coordinate system. For an intrinsic definition of the $w_{x}^{(n)}$ we may start from a map $\xi$

$$
T_{x} \stackrel{\xi}{\longrightarrow} \mathscr{M}
$$

from the tangent space at $x$ into the manifold with properties

ii)

$$
\begin{aligned}
\xi(0) & =x, \\
\left.\frac{d \xi(s z)}{d s}\right|_{s=0} & =z ; \quad z \in T_{x},
\end{aligned}
$$

i.e. the tangent vector of the parametrized curve in $\mathscr{M}$ which is the image of the ray $s z$ shall be $z$. We may then consider

$$
w_{x}^{(n)}(f)=\lim _{s \rightarrow 0} N^{(n)}(s) \int f\left(z_{1}, \ldots, z_{n}\right) \Phi\left(\xi\left(s z_{1}\right)\right) \ldots \Phi\left(\xi\left(s z_{n}\right) d \mu\left(z_{1}\right) \ldots d \mu\left(z_{n}\right),\right.
$$

where $d \mu(z)=\sqrt{-g(x)} d^{4} z$, provided there exists a normalization factor $N^{(n)}(s)$ which gives a finite, nonvanishing limit for all Laurent Schwartz test functions $f$. If such an $N^{(n)}(s)$ exists for one mapping with properties i), ii), one sees that it will be the same for any such map and the result (2.2) is independent of the choice of $\xi$. Of course, for this to hold it is assumed that $g$ is not degenerate at the point $x$.

The difference between $w_{x}^{(n)}$ calculated with the mappings $\xi$ and $\xi^{\prime}$ is

$$
\Delta=\lim _{s \rightarrow 0} N_{(s)}^{(n)} \int\left[f\left(z_{1}^{\prime} \ldots z_{n}^{\prime}\right) J-f\left(z_{1}, \ldots, z_{n}\right)\right] \Phi\left(\xi\left(s z_{1}\right)\right) \ldots \Phi\left(\xi\left(s z_{n}\right)\right) \prod d \mu\left(z_{i}\right),
$$

where $z_{i}^{\prime}=s^{-1} M s z_{i}, J=\left|\frac{\partial z^{\prime}}{\partial z}\right|$ and $M=\xi^{\prime-1} \xi$. Since $\xi$ and $\xi^{\prime}$ shall both satisfy the properties i), ii) the map $M$ from $T_{x}$ to $T_{x}$ has the form

$$
(M z)^{\mu}=z^{\mu}+a_{v e}^{\mu} z^{v} z^{\varrho}+\ldots .
$$


Thus

$$
\begin{gathered}
z_{i}^{\mu^{\prime}}=z_{i}^{\mu}+s a_{v \varrho}^{\mu} z_{i}^{v} z_{i}^{o}+O\left(s^{2}\right), \\
J=1+O(s) ; \quad f\left(z_{1}^{\prime}, \ldots, z_{n}^{\prime}\right) J-f\left(z_{1}, \ldots, z_{n}\right)=O(s) .
\end{gathered}
$$

Since $f\left(z_{1}^{\prime} \ldots z_{n}^{\prime}\right) J-f\left(z_{1}, \ldots, z_{n}\right)$ regarded as a function of the $z_{i}$ is again a Laurent Schwartz test function, $\Delta$ vanishes of order $s$.

Now the tangent space is naturally isomorphic to Minkowski space with distinguished origin and therefore one may expect that the set of distributions $w_{x}^{(n)}$ should have the same properties as the Wightman distributions in Minkowski space.

The first property is "Poincaré-invariance", i.e.

$$
w_{x}^{(n)}(f)=w_{x}^{(n)}\left(f^{\prime}\right)
$$

where

$$
f^{\prime}\left(z_{1} \ldots z_{n}\right)=f\left(\Lambda z_{1}+a, \ldots, \Lambda z_{n}+a\right)
$$

The translation invariance corresponds to the continuity in $x$ of $w_{x}^{(n)}$ since a common finite translation of the $z_{i}$ in tangent space corresponds to an infinitesimal shift of the base point $x$. The Lorentz-invariance follows in the generic case from the following consideration: Since the $w_{x}^{(n)}$ are numerical distributions whose specification is part of the theory there is - in the absence of external fields other than the metric field $g_{\mu \nu}$ - only one natural way to transport $w_{x}^{(n)}$ to $w_{x^{\prime}}^{(n)}$, namely by the affine connection along some path. The result has to be path independent. If the curvature is not zero, then the transport along a closed path from $x$ to $x$ produces a common Lorentz transformation of all the tangent vectors $z_{i}$. In the generic case we can obtain an arbitrary Lorentz transformation by suitably choosing a closed path.

The next essential property of the Wightman distributions is the support property in momentum space. In flat space-time it expresses the requirement that there should exist a ground state, the vacuum. In Riemannian space-time we can retain a local version of this, which we may call the principle of local stability, limiting the support of the distributions $w_{x}^{(n)}$ in momentum space (cotangent space). For the 2-point distribution this means that with

$$
w_{x}^{(2)}\left(z_{1}, z_{2}\right)=\int \tilde{w}_{x}^{(2)}(p) e^{i p\left(z_{1}-z_{2}\right)} d \mu(p),
$$

the support of $w^{(2)}$ is restricted to the forward cone

$$
p^{0} \geqq 0 ; \quad(p, p) \leqq 0,
$$

where the scalar products, of course, are defined with the local metric $g_{\mu v}(x)$.

The construction of the $w_{x}^{(n)}$ as the scaling limits in (2.2) implies one further property. We discuss it here only for the linear theory. There the commutator $\left[\Phi\left(x_{1}\right), \Phi\left(x_{2}\right)\right]$ is already a numerical valued distribution and therefore the antisymmetric part of $w_{x}^{(2)}$ is directly given. This determines the scaling factor

$$
N^{(2)}(s)=s^{2},
$$


and also the normalization. Replacing $s$ by $\lambda s$ in (2.2), we obtain the scaling property of $w^{(2)}$

$$
w_{x}^{(2)}\left(\lambda z_{1}, \lambda z_{2}\right)=\lambda^{-2} w^{(2)}\left(z_{1}, z_{2}\right),
$$

and correspondingly, since $d \mu(\lambda p)=\lambda^{4} d \mu(p)$,

$$
\tilde{w}_{x}^{(2)}(\lambda p)=\lambda^{-2} \tilde{w}_{x}(p)
$$

This scaling property implies that the value of the mass disappears. Any finite mass value is scaled down to zero.

Taking all the properties together we get

$$
w_{x}^{(2)}\left(z_{1}, z_{2}\right)=D_{x}^{(+)}\left(z_{1}-z_{2}\right)=(2 \pi)^{-2}\left(g_{\mu v}(x) z^{\mu} z^{v}\right)^{-1},
$$

where $z=z_{1}-z_{2}$ and $z^{0}$ is given an infinitesimal negative imaginary part, i.e. $z^{0}$ is replaced by $z^{0}-i \varepsilon$.

There is one further important property of the set of Wightman distributions. They should define a positive linear form on the tensor algebra of test functions. We should first note that the passage to tangent space by (2.2) is not the limit of an automorphism group of the observable algebra. In fact it is not even a mapping from this algebra to the numbers since the same algebraic element may be described by many different $\Phi(f)$ due to the field equations. However, if we choose a space-like surface and express each element in the observable algebra by the Cauchy data $\Phi, \frac{\partial \Phi}{\partial \mathrm{x}^{0}}$ on this surface, then the observable algebra of a neighborhood of a point on this space-like surface is mapped into the "kinematical algebra" generated by the $\Phi, \frac{\partial \Phi}{\partial x^{0}}$ on this surface and (2.2) defines a positive map from this algebra to the tensor algebra of test functions in tangent space. The consequences of this positivity are that with $w_{x}^{(2)}$ given by $(2.10)$ and $w_{x}^{(1)}=0$, the extension to the higher $w_{x}^{(n)}$ is unique. The truncated (correlated) parts vanish for $n>2$

$$
w_{x}^{(n) T}=0 \text { for } n>2,
$$

i.e. the set of $w_{x}^{(n)}$ is precisely equal to the vacuum expectation values of a massless free field in Minkowski space.

One further remark is needed for the discussion in the next section. If we have a coordinate patch whose boundary is a horizon so that the metric becomes degenerate there in the coordinates used we can still check in this coordinate system whether a state is allowed by the local definiteness condition on the boundary, because for space-like $z_{1}-z_{2}$ the distribution $w^{(2)}\left(z_{1}, z_{2}\right)$ is a continuous function which may be computed from $\left\langle\Phi\left(x_{1}\right) \Phi\left(x_{2}\right)\right\rangle$ with $x_{1}$ moving towards the boundary from the inside of the patch.

\section{Horizons and Hawking Temperature}

The simplest example of a horizon has been given by Rindler [5]. Considering the subset of Minkowski space (right wedge $W_{r}$ )

$$
x^{1} \geqq\left|x^{0}\right|,
$$


we may coordinatize it by $\left(\tau, \varrho, x^{\perp}\right)$ related to the Minkowski coordinates by

$$
\begin{aligned}
x^{0} & =\varrho \sinh \tau, \\
x^{1} & =\varrho \cosh \tau, \\
x^{2}, x^{3} & =x^{\perp} .
\end{aligned}
$$

The hyperhalfplanes in Minkowski space

$$
H^{(+)}: x^{0}=x^{1} \geqq 0 ; \quad H^{(-)}:-x^{0}=x^{1} \geqq 0
$$

are the future (respectively past) horizons for a family of observers moving on world lines staying entirely in $W_{r}$. Namely, if such an observer sends a signal crossing $H^{(+)}$, none of his family can receive its echo back. In particular one may consider observers moving with uniform acceleration in the $x^{1}$-direction whose world lines are the hyperbolas $\varrho=$ const, $x^{\perp}=$ const. Their proper time element is given by $d s=\varrho d \tau$, the acceleration by $\varrho^{-1}$. Therefore, for such an observer $\tau$ would be a natural time coordinate and, if he used $u=\log \varrho$ instead of $\varrho$ as a spatial coordinate then the Rindler region (3.1) would be represented by all of $\mathbb{R}^{4}$ in these coordinates, i.e. the horizons would be removed to infinity. The metric is

$$
d s^{2} \equiv-\varrho^{2} d \tau^{2}+d \varrho^{2}+d x^{\perp^{2}}=e^{2 u}\left(-d \tau^{2}+d u^{2}\right)+d x^{\perp^{2}} .
$$

The essential features which this example shares with the Schwarzschild solution are the existence of a time-like isometry, a 3-parameter group of space-like isometries acting on the coordinates $x^{\perp}$ (the angles $\vartheta, \varphi$ in the Schwarzschild case) and of a horizon. Using orthogonal coordinates in which $x^{0}$ measures the coordinate along the time-like Killing vector and $x^{1}$ the approach to the horizon, the metric is of the form

$$
d s^{2}=-A d x^{0^{2}}+B d x^{1^{2}}+C g_{\alpha \beta} d x^{\alpha} d x^{\beta} ; \quad \alpha, \beta=2,3,
$$

where $A, B, C$ are functions of $x^{1}$ alone, $g_{\alpha \beta}$ are independent of $x^{0}$ and $x^{1}$ and the horizon is characterized by

$$
A\left(x^{1}\right)=0
$$

The integral

$$
\Delta x^{0}=\int^{H}\left(\frac{B}{A}\right)^{1 / 2} d x^{1}
$$

diverges so that the coordinate time $x^{0}$ taken by a signal from the inside to reach the horizon is infinite. In fact, the divergence of (3.7) at the horizon is logarithmic in the Schwarzschild- and Rindler case and the quantity

$$
\gamma=\left.B^{-1 / 2} \frac{d A^{1 / 2}}{d x^{1}}\right|_{H}
$$

is finite. This quantity, which is independent of the choice of the coordinate $x^{1}$ (within the specified conventions) and scales inversely proportional to the time coordinate is related to a distinguished temperature $T=\frac{\gamma}{2 \pi}$, the Hawking 
temperature. To arrive at this interpretation of (3.8) one considers a quantum field $\Phi$ on the Riemannian manifold (3.5). We shall show how this distinguished temperature arises from the principles of local definiteness and stability described in the last section when applied to points on the horizon ${ }^{3}$. To make the calculations analytically explicit we shall perform them in the Rindler case for a scalar quantum field satisfying the covariant Klein-Gordon-equation corresponding to (3.4)

$$
-\varrho^{-2} \frac{\partial^{2} \Phi}{\partial \tau^{2}}+\frac{\partial^{2} \Phi}{\partial \varrho^{2}}+\varrho^{-1} \frac{\partial \Phi}{\partial \varrho}+\Delta^{\perp} \Phi-m^{2} \Phi=0,
$$

and canonical commutation relations

$$
\left[\frac{\partial \Phi}{\partial \tau}\left(0, \varrho, x^{\perp}\right), \Phi\left(0, \varrho^{\prime}, x^{\perp^{\prime}}\right)\right]=-i \varrho \delta\left(\varrho-\varrho^{\prime}\right) \delta\left(x^{\perp}-x^{\perp^{\prime}}\right) .
$$

Before doing this we have to refer to results coming from an entirely different circle of ideas.

Bisognano and Wichmann [6] considered the restriction of the vacuum state in Minkowski quantum field theory to the right wedge (3.1). It was known due to the theorem of Reeh and Schlieder [7] (which follows from the assumption that the vacuum is the state of lowest energy) and from the mathematical theory of modular operators by Tomita and Takesaki [8] that for any region of Minkowski space which has a nonvoid causal complement there exists a distinguished 1-parameter group of automorphisms of the algebra of this region, the modular automorphism group associated with the vacuum state. Bisognano and Wichmann determined the modular automorphism group of the vacuum state for the right-hand wedge and found the surprising result that it could be characterized quite independently of the detailed nature of the theory in purely geometrical terms: The modular automorphism group of the restriction of the vacuum state to the right wedge is the group of Lorentz boosts in the $x^{1}-x^{0}$ plane with a scaling factor $2 \pi$. In the coordinates (3.2) it corresponds to a translation of the $\tau$-coordinate $\tau \rightarrow \tau+2 \pi s$ ( $s$ is the group parameter).

On the other hand we know that the relation between a (faithful) state and its modular automorphism group is precisely the one which prevails between an dequilibrium state and the group of time translation automorphisms [9]. Specifically, an equilibrium state at inverse temperature $\beta$ satisfies the KMScondition $^{4}$

$$
\int\left\langle B \alpha_{t}(A)\right\rangle_{\beta} e^{-i \omega t} d t=e^{\beta \omega} \int\left\langle\alpha_{t}(A) B\right\rangle_{\beta} e^{-i \omega t} d t,
$$

where \langle\rangle$_{\beta}$ denotes the expectation value in the state, $\alpha_{t}(A)$ is the time translate of $A ; B$ and $A$ are arbitrary elements of the observable algebra. This condition is synonymous with the statement that $\alpha_{\beta s}$ is the modular automorphism group of the state. Therefore the result of Bisognano and Wichmann may be interpreted as saying that for an observer in $W_{r}$ who takes $\tau$ as his time coordinate, the Minkowski vacuum state appears as an equilibrium state with temperature $(2 \pi)^{-1}$.

3 More precisely to points of the 2-dimensional manifold $H^{(+)} \cap H^{(-)}$

4 KMS stands for Kubo, Martin, Schwinger who first wrote the Gibbs canonical ensemble in a form which could be applied to an infinite medium 
Sewell [10] has pointed out the parallelism between this result and the Hawking temperature. In a subsequent paper he formulated axioms for quantum field theory on certain manifolds including the Kruskal-Schwarzschild case which served to characterize a distinguished global state on the (complete) manifold, generalizing the vacuum in Minkowski space in the Wightman frame. From the point of view described in the previous sections we would like to separate two questions. First, assume that for some reason we have an equilibrium state with respect to the time-like Killing vector field in the incomplete (Rindler or Schwarzschild) region. Then we can show that local definiteness and stability allows only one value of the temperature. The other question is: under what circumstances can we expect to find a stationary state with respect to this Killing vector field in a finite neighborhood of the horizon? This demands assumptions on the nature of the state in the past at space-like infinity $[1,12,13]$ (see also the summary at the end of this section).

Now we want to find the class of allowed states on $W_{r}$ for the quantum field theory (3.9), (3.10). In analogy to the usual procedure we can express the field in terms of "creation" - and "destruction" - operators by using a complete system of $C$-number-solutions of (3.9). One gets

$$
\Phi\left(\tau, \varrho, x^{\perp}\right)=\int_{\omega=0}^{\infty} K_{i \omega}(\mu \varrho)\left\{a\left(\omega, \mathbf{k}^{\perp}\right) e^{i\left(\mathbf{k}^{\perp} \mathbf{x}^{\perp}-\omega \tau\right)}+a^{*}\left(\omega, \mathbf{k}^{\perp}\right) e^{-i\left(\mathbf{k}^{\perp} \mathbf{x}^{\perp}-\omega \tau\right)}\right\} d \omega d^{2} k^{\perp},
$$

where $K_{i \omega}$ is the modified Hankel function with purely imaginary Index $i \omega$ and

$$
\mu=\left(\mathbf{k}^{\perp^{2}}+m^{2}\right)^{1 / 2} .
$$

If we use the normalization convention of [14 and 15] (which unfortunately is different from the one used by Wittaker and Watson), one has the orthogonality relations

$$
\int_{0}^{\infty} K_{i \omega^{\prime}}(\mu \varrho) K_{i \omega}(\mu \varrho) \frac{d \varrho}{\varrho}=\frac{\pi^{2}}{2}(\omega \sinh \pi \omega)^{-1} \delta\left(\omega-\omega^{\prime}\right),
$$

and completeness relation

$$
\int_{0}^{\infty} \omega \sinh \pi \omega K_{i \omega}\left(\mu \varrho_{1}\right) K_{i \omega}\left(\mu \varrho_{2}\right) d \omega=\frac{\pi^{2}}{2} \varrho_{1} \delta\left(\varrho_{1}-\varrho_{2}\right)
$$

(see [15] under Kontorowich-Lebedev-Transformation). Correspondingly the commutation relations (3.10) become

$$
\begin{aligned}
{\left[a\left(\omega, \mathbf{k}^{\perp}\right), a^{*}\left(\omega^{\prime}, \mathbf{k}^{\perp^{\prime}}\right)\right] } & =\left(4 \pi^{4}\right)^{-1} \sinh \pi \omega \delta\left(\omega-\omega^{\prime}\right) \delta^{2}\left(\mathbf{k}^{\perp}-\mathbf{k}^{\perp^{\prime}}\right), \\
{[a, a] } & =\left[a^{*}, a^{*}\right]=0 .
\end{aligned}
$$

The expectation value of $\Phi\left(x_{1}\right) \Phi\left(x_{2}\right)$ in a state can then be written

$$
\begin{aligned}
\langle\Phi & \left.\left(\tau_{1}, \varrho_{1}, \mathbf{x}_{1}^{\perp}\right) \Phi\left(\tau_{2}, \varrho_{2}, \mathbf{x}_{2}^{\perp}\right)\right\rangle \\
= & \int_{-\infty}^{\infty} d \omega_{1} d \omega_{2} \int d^{2} \mathbf{k}_{1}^{\perp} d^{2} \mathbf{k}_{2}^{\perp} K_{i \omega_{1}}\left(\mu_{1} \varrho_{1}\right) K_{i \omega_{2}}\left(\mu_{2} \varrho_{2}\right) \\
& \cdot \exp i\left(\mathbf{k}_{1}^{\perp} \mathbf{x}_{1}^{\perp}-\mathbf{k}_{2}^{\perp} \mathbf{x}_{2}^{\perp}-\omega_{1} \tau_{1}+\omega_{2} \tau_{2}{ }^{\prime} \sum\left(\omega_{1}, \mathbf{k}_{1}^{\perp} \mid \omega_{2}, \mathbf{k}_{2}^{\perp}\right),\right.
\end{aligned}
$$


where

$$
\begin{aligned}
\Sigma^{++} & =\left\langle a\left(\omega, \mathbf{k}^{\perp}\right) a^{*}\left(\omega^{\prime}, \mathbf{k}^{\perp}\right)\right\rangle, & & \omega, \omega^{\prime}>0, \\
\Sigma^{--} & =\left\langle a^{*}\left(|\omega|,-\mathbf{k}^{\perp}\right) a\left(\left|\omega^{\prime}\right|,-\mathbf{k}^{\perp>}\right\rangle,\right. & & \omega, \omega^{\prime}<0, \\
\Sigma=\Sigma^{+-} & =\left\langle a\left(\omega, \mathbf{k}^{\perp}\right) a\left(\left|\omega^{\prime}\right|,-\mathbf{k}^{\perp}\right\rangle,\right. & & \omega>0, \quad \omega^{\prime}<0, \\
\Sigma^{-+} & =\left\langle a^{*}\left(|\omega|,-\mathbf{k}^{\perp}\right) a^{*}\left(\omega^{\prime}, \mathbf{k}^{\perp}\right)\right\rangle, & & \omega<0, \quad \omega^{\prime}>0 .
\end{aligned}
$$

The commutation relations (3.15) impose the restriction

$$
\begin{aligned}
& \Sigma^{++}\left(\omega, \mathbf{k}^{\perp} \mid \omega^{\prime}, \mathbf{k}^{\perp}\right)-\Sigma^{--}\left(-\omega^{\prime},-\mathbf{k}^{\perp} \mid-\omega,-\mathbf{k}^{\perp}\right) \\
& \quad=\left(4 \pi^{4}\right)^{-1} \sinh \pi \omega \delta\left(\omega-\omega^{\prime}\right) \delta^{(2)}\left(\mathbf{k}^{\perp}-\mathbf{k}^{\perp}\right) .
\end{aligned}
$$

We shall discuss the additional restrictions on the state arising from the conditions in tangent space only for the case of "equilibrium states" with respect to the "time translation"

$$
\alpha_{\tau^{\prime}} \Phi\left(\tau, \varrho, \mathbf{x}^{\perp}\right)=\Phi\left(\tau+\tau^{\prime}, \varrho, \mathbf{x}^{\perp}\right) .
$$

The KMS-condition (3.11) allows us then to express the expectation value of a product by that of a commutator

$$
\langle A B\rangle_{\beta}=(2 \pi)^{-1} \int_{-\infty}^{\infty} \frac{e^{\beta \omega}}{e^{\beta \omega}-1}\left\langle\left[\alpha_{\tau}(A), B\right]\right\rangle_{\beta} e^{i \omega \tau} d \omega d \tau .
$$

For the left-hand side of (3.17) this gives

$$
\left\langle\Phi\left(\tau_{1}, \mathbf{x}_{1}\right) \Phi\left(\tau_{2}, \mathbf{x}_{2}\right)\right\rangle_{\beta}=\frac{i}{2 \pi} \int_{-\infty}^{\infty} \Delta\left(\tau_{1}+\tau, \mathbf{x}_{1} \mid \tau_{2}, \mathbf{x}_{2}\right) \frac{e^{\beta \omega}}{e^{\beta \omega}-1} e^{+i \omega \tau} d \tau d \omega,
$$

with

$$
\begin{aligned}
\mathbf{x}_{i} & =\left(\varrho_{i}, x_{i}^{\perp}\right), \\
i \Delta\left(x_{1} \mid x_{2}\right) & =\left[\Phi\left(x_{1}\right), \Phi\left(x_{2}\right)\right] .
\end{aligned}
$$

By (3.12), (3.16) we have in the Rindler case

$$
\begin{aligned}
& \text { if } \Delta\left(\tau_{1}+\tau, \mathbf{x}_{1} \mid \tau_{2}, \mathbf{x}_{2}\right) e^{i \omega \tau} d \tau=\left(2 \pi^{3}\right)^{-1} \sinh \pi \omega e^{-i \omega\left(\tau_{1}-\tau_{2}\right)} \\
& \cdot \int K_{i \omega}\left(\mu \varrho_{1}\right) K_{i \omega}\left(\mu \varrho_{2}\right) e^{i \mathbf{k}^{\perp}\left(\mathbf{x}_{1}^{\frac{1}{1}}-\mathbf{x}_{2}^{\frac{1}{2}}\right)} d^{2} \mathbf{k}^{\perp} .
\end{aligned}
$$

We should put

$$
\tau_{2}-\tau_{1}=s z^{0}, \quad \varrho_{2}-\varrho_{1}=s z^{1}, \quad \mathbf{x}_{2}^{\perp}-\mathbf{x}_{1}^{\perp}=s \mathbf{z}^{\perp}
$$

and evaluate the singularity as $s=0$.

If $x_{1}$ is an inner point of the Rindler region, i.e. $\varrho_{1}>0$, we see that in the integral over $\omega$ and $\mathbf{k}^{\perp}$ which remains after inserting (3.24), (3.25) into (3.22) finite ranges of $\omega$ and $\mathbf{k}^{\perp}$ give no singularity in $s$, only the asymptotic part for large $\omega$ and large $\mathbf{k}^{\perp}$ is relevant in the limit. In that region, however, the Bose factor $\frac{e^{\beta \omega}}{e^{\beta \omega}-1}$ becomes independent of $\beta$, namely 1 for $\omega \rightarrow+\infty$ and 0 for $\omega \rightarrow-\infty$ for positive values of $\beta$ and $\mu$ can be replaced by $\left|\mathbf{k}^{\perp}\right|$. Therefore, for any (positive) value of $\beta$ the singular 
part of (3.22) will be precisely the vacuum expectation value of a massless field as demanded in (2.10). Thus (2.10) applied to the inner point of the Rindler wedge allows all positive temperatures.

If we know that the horizon is in the interior of an extended manifold, then we have to demand (2.10) also for the points of the horizon. As $\varrho_{1} \rightarrow 0$ the commutator function $\Delta$ becomes more and more extended in $\tau$ and its Fourier transform with respect to $\tau$ becomes concentrated around $\omega=0$. This can be seen from the form of $K_{i \omega}\left(\mu \varrho_{1}\right)$ for small argument

$$
K_{i \omega}\left(\mu \varrho_{1}\right) \approx \frac{\pi i}{2 \sinh \pi \omega}\left(\frac{e^{i \omega \log \frac{\mu \varrho_{1}}{2}}}{\Gamma(1+i \omega)}-\frac{e^{-i \omega \log \frac{\mu \varrho_{1}}{2}}}{\Gamma(1-i \omega)}\right) .
$$

As $\varrho_{1} \rightarrow 0, \log \varrho_{1} \rightarrow-\infty$, and this becomes equivalent to

$$
K_{i \omega}\left(\mu \varrho_{1}\right) \rightarrow \lim _{\kappa \rightarrow \infty} \frac{\sin \kappa \omega}{\omega}=\pi \delta(\omega) .
$$

In the limit $\varrho_{1}=0$ the expression (3.22) with the insertion of (3.24), (3.25), (3.27) becomes

$$
\begin{gathered}
\left\langle\Phi\left(\tau_{1}, 0, \mathbf{x}_{1}^{\perp}\right) \Phi\left(\tau_{2}, \varrho_{2}, \mathbf{x}_{2}^{\perp}\right)\right\rangle_{\beta}=\left(4 \pi^{2} \beta\right)^{-1} \int K_{0}\left(\mu s z^{1}\right) e^{-i \mathbf{k}^{\perp} \mathbf{s}^{\perp}} d^{2} \mathbf{k}^{\perp} \\
\left.\underset{s \rightarrow 0}{\longrightarrow} \int_{0}^{\infty}\left(2 \pi \beta s^{2}\right)^{-1} K_{0}\left(k^{\prime} z^{1}\right) J_{0}\left(k^{\prime} \mid \mathbf{z}^{\perp}\right)\right) k^{\prime} d k^{\prime}=\left(2 \pi \beta s^{2}\right)^{-1}\left(z^{1^{2}}+\mathbf{z}^{\perp^{2}}\right)^{-1} .
\end{gathered}
$$

Here we have changed from $\left|\mathbf{k}^{\perp}\right|$ to $k^{\prime}=s\left|\mathbf{k}^{\perp}\right|$, replaced $s \mu=\left(k^{\prime 2}+s^{2} m^{2}\right)^{1 / 2}$ by $k^{\prime}$. For the last evaluation see $[14$, p. 37]. Thus on the horizon

$$
\left\langle w^{(2)}\right\rangle_{\beta}=(2 \pi \beta)^{-1}\left(z^{2}\right)^{-1},
$$

and comparison with (2.10) shows that this agrees with the principles of local definiteness and stability if and only if

$$
\beta=2 \pi \text {. }
$$

Let us consider the more general case (3.5). The covariant Klein-Gordon equation is now

$$
-A^{-1} \frac{\partial^{2} \Phi}{\partial x^{0^{2}}}+B^{-1} \frac{\partial^{2} \Phi}{\partial x^{1^{2}}}+D \frac{\partial \Phi}{\partial x^{1}}+C^{-1} \Delta^{\perp} \Phi-m^{2} \Phi=0,
$$

where

$$
D=B^{-1} \frac{d}{d x^{1}} \log \left(C A^{1 / 2} B^{-1 / 2}\right),
$$

and $\Delta^{\perp}$ is the Laplacian in the transversal coordinates with respect to the metric $g_{\alpha \beta}$ $(\alpha, \beta=2,3)$. To compute the expectation value $\left\langle\Phi\left(x_{1}\right) \Phi\left(x_{2}\right)\right\rangle$ in a KMS-state (Gibbs state) for inverse temperature $\beta$ with respect to the time translation $x^{0} \rightarrow x^{0}+t$ we start from (3.21) [or (3.22) in the adapted notation]. We need only the commutator $\Delta\left(x_{1} \mid x_{2}\right)$ defined by (3.23). This distribution is uniquely determined by the fact that it is a solution of the wave equation (3.31) in both $x_{1}$ 
and $x_{2}$, vanishes for equal times $x_{1}^{0}=x_{2}^{0}$ and is normalized by

$$
\left.\frac{\partial}{\partial x_{2}^{0}} \Delta\left(x_{1} \mid x_{2}\right)\right|_{x_{1}^{0}=x_{2}^{0}}=\delta_{g}^{(3)}\left(x_{1} \mid x_{2}\right)
$$

where $\delta_{g}^{(3)}$ is the "covariant $\delta$-function" on the hyperplane $x_{1}^{0}=x_{2}^{0}$ defined by the properties

$$
\begin{gathered}
\delta_{g}^{3}\left(x_{1} \mid x_{2}\right)=0 \text { for } x_{1} \neq x_{2} \\
\int \delta_{g}^{3}\left(x_{1} \mid x_{2}\right) C B^{1 / 2} A^{-1 / 2}\left|g_{\alpha \beta}\right|^{1 / 2} \prod d x_{2}^{i}=1
\end{gathered}
$$

Separation of the variables gives

$$
i \Delta\left(x_{1} \mid x_{2}\right)=\int d \omega \operatorname{S}_{\kappa} R_{\omega, \kappa}\left(x_{1}^{1}, x_{2}^{1}\right) P_{\kappa}\left(\mathbf{x}_{1}^{\perp}, \mathbf{x}_{2}^{\perp}\right) e^{-i \omega\left(x_{1}^{0}-x_{2}^{0}\right)},
$$

where $R_{\omega, \kappa}$ is a solution of the radial wave equation

$$
\left\{\mathrm{B}^{-1} \frac{\mathrm{d}^{2}}{\mathrm{dx}^{1^{2}}}+D \frac{d}{d x^{1}}-C^{-1} \kappa^{2}-m^{2}+A^{-1} \omega^{2}\right\} R=0
$$

in both $x_{1}^{1}$ and $x_{2}^{1} \cdot \kappa^{2}$ runs through the spectrum of $-\Delta^{\perp}$ and $P_{\kappa}$ is the spectral projector. Specifically

$$
\mathrm{S}_{\kappa} \ldots P_{\kappa}=\sum_{\ell, m} \ldots Y_{\ell}\left(\vartheta_{1}, \varphi_{1}\right) \bar{Y}_{\ell}^{m}\left(\vartheta_{2}, \varphi_{2}\right) ; \quad \kappa^{2}=\ell(\ell+1)
$$

in the Schwarzschild case where the transversal manifold is a 2-sphere and

$$
\underset{\kappa}{\mathrm{S}} \ldots P_{\kappa}=(2 \pi)^{-2} \int \ldots e^{i \mathbf{k}^{\perp}\left(\mathbf{x}_{1}^{\perp}-\mathbf{x}_{\frac{1}{2}}^{\perp}\right)} d^{2} \mathbf{k}^{\perp} ; \quad \kappa^{2}=\mathbf{k}^{\perp^{2}}
$$

in the Rindler case where the transverse manifold is a 2-plane. As one might expect the difference between the two cases (3.37), (3.38) disappears when one passes to the tangent space in the transversal coordinates. Explicitly, for $\mathbf{x}_{1}^{\perp}-\mathbf{x}_{2}^{\perp}$ only the very large values of $\kappa$ are relevant and, in the case (3.37)

$$
\sum Y_{\ell}^{m}\left(\vartheta_{1}, \varphi_{1}\right) \bar{Y}_{\ell}^{m}\left(\vartheta_{2}, \varphi_{2}\right)=\frac{2 \ell+1}{4 \pi} P_{\ell}\left(\cos \vartheta_{12}\right) \rightarrow \frac{\ell}{2 \pi} J_{0}\left(\ell \vartheta_{12}\right),
$$

when $\ell \rightarrow \infty$ such that $\ell \vartheta_{12}$ remains fixed. The summation $\sum_{\ell}$ can then be replaced by $\int d \ell$, and we obtain

$$
\mathrm{S}_{\kappa} \ldots P_{\kappa}=(2 \pi)^{-1} \int \ldots \kappa J_{0}\left(\kappa\left|\mathbf{x}^{\perp}\right|\right) d \kappa
$$

where $\left|\mathbf{x}^{\perp}\right|=\left(g_{\alpha \beta} x^{\alpha} x^{\beta}\right)^{1 / 2}$. This is the same expression as one obtains from (3.38) and which was used in (3.28).

With the ansatz (3.35) the normalization for the radial part of $\Delta$ becomes

$$
\int \omega R_{\omega, \kappa}\left(x_{1}^{1}, x_{2}^{1}\right) d \omega=A^{1 / 2} B^{-1 / 2} C^{-1} \delta\left(x_{1}^{1}-x_{2}^{1}\right) .
$$

We must now specify the conditions for the functions $A, B, C$ determining the metric. As mentioned earlier the horizon is characterized by $A=0$ and we require

$$
\left.B^{-1 / 2} \frac{d A^{1 / 2}}{d x^{1}}\right|_{H}=\gamma \quad \text { (finite, } \neq 0 \text { ). }
$$


We add

$$
\left.C\right|_{H}=\approx C_{0}(\text { finite }, \neq 0) ;\left.\quad B^{-1 / 2} \frac{d C}{d x^{1}}\right|_{H}=0 .
$$

These conditions are independent of the choice of the $x^{1}$-coordinate (apart from the required orthogonality to $x^{0}, x^{\perp}$, (which latter are fixed by the Killing vector fields. Under these conditions $C$ may be replaced by the constant $C_{0}$ for the subsequent discussion where only the neighborhood of the horizon enters in the radial wave equation (3.36) and the normalization (3.41). The constant $C_{0}$ can be absorbed by a rescaling of $\kappa$. Putting

$$
C_{0}^{-1 / 2} \kappa=k ; \quad C_{0} R_{\omega, C_{0}^{1 / 2} k}=S_{\omega, k},
$$

we get from (3.22), (3.35), (3.40) (for small $\mathbf{x}_{1}^{\perp}-\mathbf{x}_{2}^{\perp}$ )

$$
\begin{aligned}
\left\langle\Phi\left(x_{1}\right) \Phi\left(x_{2}\right)\right\rangle_{\beta}= & (2 \pi)^{-1} \int_{-\infty}^{\infty} d \omega \int_{0}^{\infty} d k \frac{e^{\beta \omega}}{e^{\beta \omega}-1} S_{\omega, k}\left(x_{1}^{1}, x_{2}^{1}\right) \\
& \cdot k J_{0}\left(k C_{0}^{1 / 2}\left|\mathbf{x}^{\perp}\right|\right) e^{-i \omega\left(x_{1}^{0}-x_{2}^{0}\right)} .
\end{aligned}
$$

The discussion can now be reduced to the one in the Rindler case by choosing the coordinate $x^{1}=\varrho$ so that $B=1$, and thus by (3.42) $A=\gamma^{2} \varrho^{2}$ near the horizon. The radial equation which $S$ has to satisfy for both $\varrho_{1}$ and $\varrho_{2}$ becomes

$$
\left(\frac{d^{2}}{d \varrho^{2}}+\frac{1}{\varrho} \frac{d}{d \varrho}-\left(k^{2}+m^{2}\right)+\frac{\omega^{2}}{\gamma^{2} \varrho^{2}}\right) S=0 .
$$

The normalization condition:

$$
\int \omega S_{\omega, k}\left(\varrho_{1}, \varrho_{2}\right) d \omega=\gamma \varrho_{1} \delta\left(\varrho_{1}-\varrho_{2}\right) .
$$

The explicit solution (neglecting the mass which is irrelevant in the limit $k \rightarrow \infty$ ):

$$
S_{\omega, k}\left(\varrho_{1}, \varrho_{2}\right)=\left(\gamma \pi^{2}\right)^{-1} \sinh \frac{\pi \omega}{\gamma} K_{i \omega / \gamma}\left(k \varrho_{1}\right) K_{i \omega / \gamma}\left(k \varrho_{2}\right)
$$

[see the completeness relation for the modified Hankel-functions (3.15)]. As $\varrho_{1}$ moves towards the horizon we obtain the limit (see 3.27)

$$
\lim _{\varrho_{1} \rightarrow \infty} \omega^{-1} S_{\omega, \kappa}\left(\varrho_{1}, \varrho_{2}\right)=\gamma^{-1} \delta(\omega) K_{0}\left(k \varrho_{\kappa}\right),
$$

and (3.45) reduces to

$$
\begin{aligned}
\left\langle\Phi(0) \Phi\left(x_{2}\right)\right\rangle_{\beta} & =(2 \pi \beta \gamma) \cdot 1 \int_{0}^{\infty} K_{0}\left(k \varrho_{2}\right) J_{0}\left(k C_{0}^{1 / 2}\left|\mathbf{x}^{\perp}\right|\right) k d k \\
& =(2 \pi \beta \gamma)^{-1}\left(\varrho_{2}^{2}+C_{0}\left|x^{\perp}\right|^{2}\right)^{-1} .
\end{aligned}
$$

Note that the transition to tangent space just needs a rescaling of $k$, i.e. the change of the integration variable in (4.50) to $k^{\prime}=s k$, in agreement with the previous claim that only very large $k$-values are relevant. Equation (3.50) agrees with the demanded form (2.10) for the metric (3.5) on the horizon precisely if

$$
\beta=2 \pi \gamma^{-1}=4 \pi(B A)^{1 / 2}\left(\frac{d A}{d x^{1}}\right)^{-1} .
$$


The physical picture becomes somewhat more transparent if one chooses $x^{1}$ so that the horizon moves to $-\infty$. This corresponds to the choice $A=B$. In the Schwarzschild case this is achieved by the tortoise coordinate

$$
x^{1}=\hat{r}=r+r_{0} \log \left(\frac{r}{r_{0}}-1\right) .
$$

The region "near the horizon" is then $\hat{r}<0$ and in this region, by (3.42) $A=e^{2 \gamma \hat{r}}$. The radial equation near the horizon is

$$
\left(\frac{d^{2}}{d \hat{r}^{2}}-e^{2 \gamma \hat{r}}\left(k^{2}+m^{2}\right)+\omega^{2}\right) S=0
$$

and the normalization

$$
\int d \omega S_{\omega, k}\left(\hat{r}_{1}, \hat{r}_{2}\right)=\delta\left(\hat{r}_{1}-\hat{r}_{2}\right) .
$$

Far away from the horizon, for $\hat{r}>r_{0}, A \rightarrow 1$, and $C^{-1} \rightarrow 0$. The effective potential has the shape pictured in Fig. 1 . The two regions $\hat{r}<0$ and $\hat{r} \gg r_{0}$ are separated by a potential barrier whose height is proportional to $k^{2}$. Since in the reduction to transversal tangent space only the behavior for $k \rightarrow \infty(\ell \rightarrow \infty)$ matters we may ignore the region $\hat{r} \gg r_{0}$ for our purpose and consider only Eq. (3.53), (3.54). This means that all incoming waves from $r=\infty$ are shielded by the centrifugal barrier from exerting an influence on our condition in the tangent space at the horizon. The determination of $S$ from (3.53), (3.54) and $S_{\omega, k}=-S_{-\omega, k}$ (resulting from the antisymmetry of $\Delta$ ) amounts then to a discussion of the scattering of a particle coming from the left in the potential of Fig. 1 by Schrödinger wave mechanics. We shall omit this discussion. However, we note that for $\omega>m$ there are in the Schwarzschild case - in contrast to the Rindler case - two linearly independent admissible solutions $R^{L}, R^{R}$ of the radial equation and, correspondingly, two sets of destruction operators $a_{\ell, m}^{L}(\omega), a_{\ell, m}^{R}(\omega)$, where the symbol $L$ denotes incoming waves from the horizon, $R$ denotes incoming waves from $r=\infty$. Characterizing a stationary, rotationally symmetric, quasifree state by the occupation number distributions $\varrho^{i j}$ :

$$
\left\langle a_{t m}^{*^{i}}(\omega) a_{\ell^{\prime} m^{\prime}}^{j}\left(\omega^{\prime}\right)\right\rangle=\delta_{\ell \ell^{\prime}} \delta_{m m^{\prime}} \delta\left(\omega-\omega^{\prime}\right) \varrho^{i j}(\omega) ; \quad i, j=L, R,
$$

we see that the tagent space condition for inner points demands

$$
\lim _{\omega \rightarrow \infty} \varrho_{b}^{i j}(\omega)=0,
$$

and for points on the horizon it gives only a condition for $\varrho^{L L}$ at $\omega=0$ which fixes the value of the Hawking temperature.

Summarizing the results of this section: We considered a (linear) quantum field on a Riemannian space with a time-like Killing vector field and a horizon which can be described by the metric (3.5) with conditions (3.42), (3.43). We showed that there is a unique temperature

$$
T=\gamma(2 \pi)^{-1}
$$

for which an "equilibrium state" with respect to the time-like Killing vector field satisfies the conditions of local definiteness and stability on the horizon. From the 


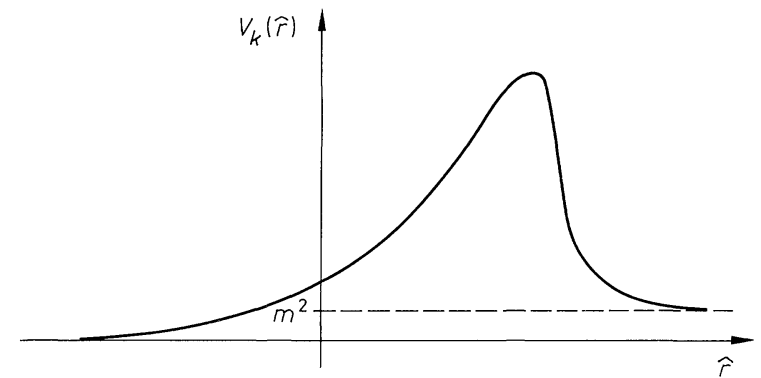

Fig. 1

computation it appears that the existence of the space-like symmetries assumed in (3.5) is needed only to the extent that $A$ and $B$ must be functions of $x^{1}$ alone near the horizon. The results of Bisognano and Wichmann for the Rindler wedge indicate that (3.55) remains valid also in the case of an interacting quantum field. But to show this we would have to understand the consequences of local definiteness and stability for the $W^{*}$-algebras of finite regions better.

It must be stressed that our discussion does not replace a detailed discussion of the history of black hole formation because every admissible state of the quantum field will look like a thermal state with the Hawking temperature (3.55) in the infinitesimal neighborhood of the horizon. This corresponds to the statement in Minkowski space that every admissible state looks like the vacuum in a sufficiently small neighborhood of a point (since the energy density is finite). In general there is no reason why the state in a finite region should be (or ultimately become) stationary with respect to a Killing vector field of the metric (if there is one). Considering the tendency to local equilibrium in Minkowski space due to interactions as described by the Boltzmann equation the relevant vector field is the time flow in the rest system of the local matter distribution and not the Killing vector field of Rindler boosts. Equilibrium states at finite temperature are not stationary with respect to the latter, only the ground state is. In a Riemannian manifold the corresponding statement would be that thermalization in the Boltzmann sense occurs with respect to a time-like vector field of geodesic flow. The KMS-state with respect to the Killing vector field in the Schwarzschild case with temperature (3.55) is a state of specially high symmetry, analoguous to the ground state in Minkowski space. In the gravitational collapse of a star we have a well defined "incoming vacuum" of the $\Phi$-field because in the past the mass distribution extended beyond the Schwarzschild radius and the metric was regular. It can be idealized as (space-like) asymptotically flat and asymptotically stationary at $t \rightarrow-\infty$ in the complete manifold. The adiabatic transform of this state when the radius of the star shrinks below the Schwarzschild radius is expected to become the "half thermal" state in which $\varrho^{L L}$ is the equilibrium distribution with the Hawking temperature and $\varrho^{R R}=0$.

\section{Local Vacuum and Particles in Nonstationary Metric}

Let us consider as the simplest example the Robertson-Walker metric

$$
d s^{2}=-A\left(x^{0}\right) d x^{0^{2}}+B\left(x^{0}\right) g_{i k} d x^{i} d x^{k} ; \quad i, k=1,2,3,
$$


where the space part is maximally symmetric (homogenous, isotropic). Thus the $x^{i}$ may be taken as three angular coordinates $\alpha, \vartheta, \varphi$ for points on a 3-sphere and

$$
d s_{r}^{2} \equiv g_{i k} d x^{i} d x^{k}=d \alpha^{2}+\sin ^{2} \alpha\left(d \vartheta^{2}+\sin ^{2} \vartheta d \varphi^{2}\right)
$$

is invariant under the group $\mathrm{SO}(4)$, the rotations of this sphere.

We want to discuss a scalar quantum field $\Phi$ satisfying the covariant KleinGordon equation in this metric:

$$
-A^{-1} \frac{\partial^{2} \Phi}{\partial x^{0^{2}}}-A^{-1} \frac{d\left(\log B^{3 / 2} A^{-1 / 2}\right)}{d x^{0}} \frac{\partial \Phi}{\partial x^{0}}+B^{-1} \Delta^{*} \Phi-m^{2} \Phi=0,
$$

where $\Delta^{*}$ is the Laplace-Beltrami operator of the 3-sphere. Using a complete system of $c$-number solutions of this equation the quantum field may be written $\left(t=x^{0}, \mathbf{x}=\alpha, \vartheta, \varphi\right)$

$$
\left.\Phi(x)=\sum_{k, \ell, m} a_{k, \ell, m} T_{k}(t) \mathscr{Y}_{k, \ell, m} \mathbf{x}\right)+a_{k, \ell, m}^{*} \bar{T}_{k}(t) \overline{\mathscr{Y}}_{k, \ell, m}(\mathbf{x}) .
$$

Here $\mathscr{Y}_{k, \ell, m}$ shall denote the normalized spherical harmonics of the 3-sphere, $T_{k}$ and $\bar{T}_{k}$ are complex conjugate solutions of the ordinary differential equation

with

$$
\frac{d^{2} T_{k}}{d t^{2}}+D(t) \frac{d T_{k}}{d t}+\omega_{k}^{2}(t) T_{k}=0,
$$

$$
\omega_{k}^{2} \equiv A B^{-1} k(k+2)+m^{2} A ; \quad D=\frac{d}{d t} \log B^{3 / 2} A^{-1 / 2} .
$$

Usually one puts $A=1$, and

$$
R(t)=B^{1 / 2}
$$

may be interpreted as the instantaneous radius of the universe. To make the formulas slightly more transparent we choose the time coordinate so that $D=0$ in (4.5) putting

$$
A=B^{3}=R^{6} \text {. }
$$

The covariant canonical commutation relations for the field give then

$$
\left[a_{k \ell m}, a_{k^{\prime} \ell^{\prime} m^{\prime}}^{*}\right]=\delta_{k k^{\prime}} \delta_{\ell \ell^{\prime}} \delta_{m m^{\prime}} ; \quad[a, a]=\left[a^{*}, a^{*}\right]=0
$$

if we normalize $T_{k}$ so that the Wronski determinant

$$
\frac{d T_{k}}{d t} \bar{T}_{k}-T_{k} \frac{d \bar{T}_{k}}{d t}=-i
$$

If we choose an arbitrary pair of complex conjugate solutions of (4.5), normalized by (4.10), then any other such pair $T_{k}^{\prime}, \bar{T}_{k}^{\prime}$ is given by

where

$$
T_{k}^{\prime}=\alpha_{1}^{k} T_{k}+\alpha_{2}^{k} \bar{T}_{k},
$$

$$
\begin{gathered}
\alpha_{1}^{k}=\cosh \lambda_{k}+i c_{k} \lambda_{k}^{-1} \sinh \lambda_{k} ; \quad \alpha_{2}^{k}=\left(a_{k}-i b_{k}\right) \lambda_{k}^{-1} \sinh \lambda_{k} \\
\lambda_{k}=\left(a_{k}^{2}+b_{k}^{2}-c_{k}^{2}\right)^{1 / 2} ; \quad a, b, c \text { real }
\end{gathered}
$$


Equations (4.11), (4.12) define a 3-parameter group of transformations for the fundamental system $T_{k}, \bar{T}_{k}$ of solutions of the harmonic oscillator with time dependent frequency (4.5). Corresponding to this we have a 3-parametric subgroup of Bogolubov transformations of the "creation-destruction-operators" $a_{k \ell m}, a_{k \ell m}^{*}$ in (4.4), and the question is whether among the possible choices for $T_{k}, \bar{T}_{k}$ (and $a_{k \ell m}, a_{k \ell m}^{*}$ ) there exists one which is physically distinguished. If the frequency $\omega_{k}$ is constant, then the choice

$$
T_{k}=\left(2 \omega_{k}\right)^{-1 / 2} e_{k}^{-i \omega t} ; \quad \bar{T}_{k}=\left(2 \omega_{k}\right)^{-1 / 2} e^{i \omega_{k} t}
$$

is so obvious that everybody takes it without argument. It is the only one which keeps positive and negative frequencies separated and (related to this) whose logarithmic derivative does not oscillate. If $\omega_{k}$ is not constant then the magnitude of the dimensionless quantity

$$
\gamma_{k}=\omega_{k}^{-2} \frac{d \omega_{k}}{d t}
$$

is relevant. Unfortunately, for almost all conceivable physical applications this quantity is so extremely small that at most an adiabatic first order correction to (4.13) may be of interest and even this only if we consider the solution for time intervals $\Delta t$ in which $\omega_{k}^{-1} \frac{d \omega_{k}}{d t} \Delta t$ becomes significant, i.e. a time period in which $\omega_{k}$ has changed significantly. In a perturbation calculation with respect to $\gamma_{k}$ starting from the zero order choice (4.13) the functions $T_{k}$ are uniquely determined. By the normalization condition (4.10) we have

$$
T_{k}=\left(2 \frac{d \sigma_{k}}{d t}\right)^{-1 / 2} e^{-i \sigma_{k}}
$$

and $\sigma_{k}$ is given by

$$
\frac{d \sigma_{k}}{d t}=\omega_{k}-\frac{1}{4} \frac{d \gamma_{k}}{d t}-\frac{1}{8} \omega_{k} \gamma_{k}^{2}+O\left(\gamma_{k}^{4}\right) .
$$

The first order adiabatic expression replacing (4.13) is just

$$
\left.T_{k}(t)=\left(2 \omega_{k}\right)^{-1 / 2} \exp -i \int_{t_{0}}^{t} \omega_{k}\left(t^{\prime}\right) d t^{\prime}\right] .
$$

We draw the following conclusions: As long as $\gamma \ll 1$ there is a clearcut definition of the vacuum state and of local particle numbers. The (Heisenberg) state annihilated by all $a_{k \ell m}$, where $T_{k}$ is given by (4.15), (4.16) may be called the vacuum. Local positive observables with vanishing vacuum expectation values may then be considered as detectors with the help of which a local particle interpretation can be built in analogy with the procedure in Minkowski space. If $\omega_{k}$ changes so fast that $\gamma_{k} \approx 1$, no meaningful definition of a vacuum seems possible and the concept of particles looses any relevance. However, this regime is hardly of interest in the Robertson-Walker universe where $\gamma \approx\left(\omega t_{0}\right)^{-1}\left(t_{0}\right.$ the age of the universe and $\left.\omega^{-1} \sim 10^{-20} \mathrm{~s}\right)$. 
Remarks. The operator

$$
\hat{H}(t)=\sum \frac{d \sigma_{k}(t)}{d t} a_{k \ell m}^{*} a_{k \ell m}
$$

with $a, a^{*}$ referring to the choice of $T_{k}$ given by (4.15), (4.16) may be regarded as the "adiabatic (time dependent) Hamiltonian." There is a common, time independent, ground state of all $\hat{H}(t)$, the physical vacuum. One checks that this state satisfies the tangent space condition of Sect. II. In this computation we replace the spherical harmonics by plane waves (see Sect. III) and obtain with

$$
\begin{gathered}
\left\langle a_{k \ell m} a_{k^{\prime} \ell^{\prime} m^{\prime}}^{*}\right)_{0}=\delta_{k k^{\prime}} \delta_{\ell \ell^{\prime}} \delta_{m m^{\prime}} \\
\lim _{s \rightarrow 0} s^{2}\langle\Phi(x+s z) \Phi(x)\rangle_{0}=\lim (2 \pi)^{-3} s^{2} \int T_{k}\left(t+s z^{0}\right) \bar{T}_{k}(t) e^{i s \mathbf{k}} d^{3} \mathbf{k} \\
=\lim (2 \pi)^{-3} \int \frac{d^{3} \mathbf{k}^{\prime}}{2 \omega_{k^{\prime} / s} \cdot s} e^{i\left(\mathbf{k}^{\prime} \mathbf{z}-s \omega_{k^{\prime} / s^{2}}\right)} \\
=(2 \pi)^{-2} A^{-1 / 2} B^{1 / 2}\left(\mathbf{z}^{2}-A B^{-1}\left(z^{0}-i \varepsilon\right)^{2}\right)^{-1} .
\end{gathered}
$$

By our choice of time coordinate (4.8) this can also be written as $(2 \pi)^{-2}\left(B z^{2}-A\left(z^{0}-i \varepsilon\right)^{2}\right)^{-1}$, which is the demanded form. We also see that if we used any other choice $T_{k}^{\prime}$ of the time functions in (4.4) related to $T_{k}$ by (4.11), then the state which is annihilated by the corresponding "destruction operators" $a_{k \ell m}^{\prime}$ will satisfy the tangent space condition if and only if in (4.11)

$$
\lim _{k \rightarrow \infty} \alpha_{2}^{k}=0
$$

This shows that the tangent space condition does not suffice to fix the local von Neumann algebras (the quasi-equivalence class of the representation of the canonical commutation relations) because it does not prescribe how fast $\alpha_{2}^{k}$ has to go to zero as $k \rightarrow \infty$, whereas within one local quasi-equivalence class we must have that $\alpha_{2}^{k}$ decreases faster than $k^{-3 / 2}$.

An interesting example in the present context is given by the ground states of the formal time dependent Hamiltonian $H(t)$ which produces the Heisenberg equation of motion

$$
\frac{\partial \Phi(t, \mathbf{x})}{\partial t}=i[H(t), \Phi(t, \mathbf{x})] ; \quad \frac{\partial \pi(t, \mathbf{x})}{d t}=i[H(t), \pi(t, \mathbf{x})] .
$$

Note that the adiabatic Hamiltonian does not satisfy $(4.21)$, i.e. $H(t) \neq \hat{H}(t)$, but

$$
H(t)=\sum \omega_{k}(t) b_{k \ell m}^{*} b_{k \ell m}
$$

where in first order

$$
b_{k \ell m}-a_{k \ell m}=-\frac{i}{4} \gamma_{k}^{(t) a *} \underset{k \ell,-m}{ }
$$

Since

$$
\gamma_{k}(t) \underset{k \rightarrow \infty}{\longrightarrow} F(t) k^{-1}
$$


the ground state of $H(t)$ satisfies the tangent space condition but is not locally normal with respect to the vacuum nor with respect to the ground state of $H\left(t_{1}\right)$ for $t_{1} \neq t$. Therefore the operators $H(t)$ and their ground states are not physically meaningful objects.

\section{Detectors}

In [2] Unruh described the construction of idealized detectors corroborating the idea that a detector, racing with acceleration a through the vacuum in Minkowski space, will register incident quanta according to the Planck distribution with temperature $\frac{a}{2 \pi}$.

This is the experimental counterpart of the fact that the vacuum is locally a KMS-state of this temperature with respect to the proper time on the world line of the accelerated detector. It suggests the questions:

1) Which elements of the observable algebra represent ideal detectors?

2) Suppose a detector, specified by its construction procedure is represented by the algebraic element $C$ if it is placed in space-time in a certain position with, say, inertial motion. Is there a transformation law which tells us which algebraic element $C^{\prime}$ represents the same detector when it is forced to move along some other world line?

We can not offer an answer here, only a few modest remarks. Looking at the KMS-condition in the form (3.21), putting $A=B^{*}$ and $C=B^{*} B$, we see that

$$
\langle C\rangle_{\beta}=\frac{e^{-\beta \omega}}{1-e^{-\beta \omega}},
$$

if

$$
\alpha_{\tau}(B)=e^{-i \omega \tau} B,
$$

and

$$
\left[B, B^{*}\right]=1 .
$$

Thus an ideal detector, sensitive only to quanta of frequency $\omega$ is represented by $C=B^{*} B$ with $B$ satisfying (5.2) and (5.3). Of course, this has to be qualified in several ways. First, to specify an approximate position and time of sensitivity, $B$ has to belong essentially to the algebra of a specified finite region in spacetime and thus (5.2) can only approximately be realized. Secondly it can be applied only if the world line of the detector follows a Killing vector field because otherwise no automorphism $\alpha_{\tau}$ exists. If we ask for a transformation law, then we encounter one further fundamental problem coming from the nonexistence of rigid bodies in relativity. This is avoided if the extension of the detector orthogonal to the world line is practically zero. To the extent to which a compromise between these conflicting demands can be achieved the geometric description (world line) together with the approximate compliance with (5.2), (5.3) will allow us to define detectors in different states of motion.

Acknowledgements. It is a pleasure to thank Fritz Coester and Klaus Fredenhagen for helpful discussions. 


\section{References}

1. Hawking, S.W.: Particle creation by black holes. Commun. Math. Phys. 43, 199 (1975)

2. Unruh, W.G.: Notes on black-hole evaporation. Phys. Rev. D14, 870 (1976); compare also: Davies, P.C.W.: Scalar particle production in Schwarzschild and Rindler metrics. J. Phys. A8, 609 (1975)

2a.Fulling, S.A.: Nonuniqueness of canonical field quantization in Riemannian space-time. Phys. Rev. D7, 2850 (1973)

3. Haag, R., Kastler, D.: Algebraic approach to quantum field theory. J. Math. Phys. 5, 848 (1964)

4. Kastler, D.: Topics in the algebraic approach to quantum field theory, Cargèse lectures 1965, Lurçat, F. (ed.). N.Y.: Gordon and Breach 1967

5. Rindler, W.: Kruskal space and the uniformly accelerated frame. Am. J. Phys. 34, 1174 (1966)

6. Bisognano, J.J., Wichmann, E.H.: On the duality condition for a Hermitian scalar field. J. Math. Phys. 16, 985 (1975); On the duality condition for quantum fields. J. Math. Phys. 17, 303 (1976)

7. Reeh, H., Schlieder, S.: Bemerkungen zur Unitäräquivalenz von Lorentzinvarianten Feldern. Nuovo Cimento 22, 1051 (1961)

8. Takesaki, M.: Tomita's theory of modular Hilbert-algebras. Berlin, Heidelberg, New York: Springer 1970

9. Haag, R., Hugenholz, N.M., Winnink, M.: On the equilibrium states in quantum statistical mechanics. Commun. Math. Phys. 5, 215 (1967)

10. Sewell, G.L.: Relativity of temperature and the Hawking effect. Phys. Lett. 79A, 23 (1980)

11. Sewell, G.L.: Quantum fields on manifolds. Ann. Phys. (USA) 141, 201 (1982)

12. Wald, R.M.: On particle creation by black holes. Commun. Math. Phys. 45, 9 (1975)

13. Dimock, D., Kay, B.S.: Classical and quantum scattering on stars and black holes. IAMPConference, Boulder 1983

14. Magnus, W., Oberhettinger, F.: Formeln und Sätze für die speziellen Funktionen der mathematischen Physik. Berlin: Springer 1943

15. H. Bateman Manuscript Project: Tables of Integral Transforms, Vol. II. California Institute of Technology. Erdélyi, A. (ed.). New York: McGraw-Hill 1954

Communicated by R. Haag

Received January 15,1984 